\title{
Comparison of Two Methods of Laparoscopic Single Port Appendectomy and Conventional Three Port Laparoscopic Appendectomy
}

\author{
Eun Young Kim, M.D. ${ }^{1}$, Hyung Ook Kim, M.D., Ph.D. ${ }^{1}$, Hung Dai Kim, M.D., Ph.D. ${ }^{1}$, \\ Jun Ho Shin, M.D., Ph.D. ${ }^{1}$, Byung Ho Son, M.D., Ph.D. ${ }^{1}$, Won Joon Choi, M.D., Ph.D. ${ }^{2}$ \\ Departments of ${ }^{1}$ Surgery, ${ }^{2}$ Anesthesiology and Pain Medicine, Kangbuk Samsung Hospital, \\ Sungkyunkwan University School of Medicine, Seoul, Korea
}

Purpose: Acute appendicitis is a type of abdominal disease that requires emergency surgery. Over the past three decades, laparoscopic appendectomy has become the standard operation for acute appendicitis. The aim of this paper is to compare the methods of single port appendectomy and conventional appendectomy and to illustrate the advantages of single port appendectomy.

Methods: The first group of patients underwent single port appendectomy using a surgical glove (Group 1), and those in the second group underwent surgery using the Triport system (Group 2). The third group of patients underwent conventional three port surgery (Group 3). Questionnaires regarding patient satisfaction with scars were administered via telephone interview. Patient characteristics, histopathological data, postoperative data, and satisfaction score were analyzed by t-test and Pearson $x^{2}$ test.

Results: A statistically significant difference in satisfaction scores was observed among the three groups. Overall, patients in the single port group using a surgical glove would be more likely to recommend the procedure to friends and family than would patients in the other two groups. Better cosmetic results were achieved for both groups of patients who underwent laparoscopic single port appendectomy, compared to those who underwent conventional three port laparoscopic appendectomy, with statistically significant difference.

Conclusion: Laparoscopic single port appendectomy using a surgical glove is a feasible and safe procedure and shows no differences in terms of risk, such as postoperative complication, compared to a conventional three-trocar technique. Use of this method resulted in better satisfaction compared with the other two groups, illustrating its cosmetic improvement.

Key words: Laparoscopic single port appendectomy, cosmetic results, Satisfaction score, Fascial closure

\section{INTRODUCTION}

Acute appendicitis is one type of abdominal condition that requires emergency surgery. Approximately 11 of 10,000 people will experience appendicitis in their lifetime. ${ }^{1}$ Since conventional laparoscopic appendectomy using three ports was introduced in $1983,{ }^{2}$ it has become a standard method for treating acute appendicitis. Even though the results of conventional ap-

Received June 24, 2013, Revised 1st, August 9, 2013; 2nd, August 22, 2013, Accepted August 28, 2013

※ Corresponding author: Won Joon Choi

Department of Anesthesiology and Pain Medicine, Kangbuk Samsung Hospital, Sungkyunkwan University School of Medicine, 108, Pyung-dong, Jongno-gu, Seoul 110-746, Korea

Tel : +82-2-2001-2134, Fax : +82-2-2001-2131

E-mail : gimo25@hanmail.net

※ Corresponding author : Byung Ho Son

Department of Surgery, Kangbuk Samsung Hospital, Sungkyunkwan University School of Medicine, 108, Pyung-dong, Jongno-gu, Seoul 110-746, Korea

Tel : +82-2-2001-2134, Fax : +82-2-2001-2131

E-mail : dackangjung@daum.net

pendectomy are satisfying, it leaves at least three or four small skin incisions that are still visible after the operation. Patients are now more interested in the cosmetic results of surgery, so, in the 1990s, laparoscopic appendectomy using a single port was introduced. ${ }^{3}$ Multiple case studies have been published comparing these two methods. However, data regarding single port appendectomy, such as safety, postoperative pain, postoperative complications, and cosmetic results, are still under investigation.

The aim of this paper is to compare the methods of laparoscopic single port appendectomy (SP) and conventional three port laparoscopic appendectomy $(\mathrm{CP})$ for acute appendicitis in order to demonstrate the advantage of single port appendectomy.

\section{MATERIALS AND METHODS}

From March 2010 to August 2011, 799 patients with clinical and radiological diagnoses of acute appendicitis were operated on at the General Surgery Department of our hospital. Six hundred ninety-seven patients with the following conditions were excluded from this study: complicated appendicitis including 
perforated appendicitis, periappendiceal abscess, and other suspected disease based on clinical laboratory results and preoperatively taken abdominopelvic CT scan. Cases converted from laparoscopic to open during operation were also excluded. One hundred two patients were ultimately selected and assessed retrospectively. Patients were assigned to each surgeon according to duty day. The modes of operation were chosen by the surgeons. Three fully trained surgeons participated in this paper. A retrospective analysis was performed to compare patient characteristics, postoperative progression, and cosmetic results.

The first group of patients underwent single port appendectomy using a surgical glove and transabdominal fascial closure (disposable suture grasper closure device, Mediflex ${ }^{\circledR}$, USA) (Group 1). Tips of the second and fifth fingers of the surgical glove (no. 6) were cut. Two 5-mm ports (excel bladeless trocar Ethicon, U.S.A) were inserted to glove and then tied. A vertical skin incision of $1 \sim 2 \mathrm{~cm}$ was made in the umbilicus and fascia, and the peritoneum was further opened. The wound retractor (U-tractor Yoo-Won Meditech, Korea) was inserted through the layers of the umbilicus and fascia, and the surgical glove was placed over the wound retractor. Next, carbon dioxide was insufflated to keep the intraabdominal pressure $12 \mathrm{~mm} \mathrm{Hg}$, and a rigid 30-degree 12-mm scope was inserted into the $12-\mathrm{mm}$ port tied in the second finger (Fig. 1). The patient was put in the Trendelenburg position with the head down and right side up. Dissecting or grasping instruments of 5-mm diameter were introduced to the remaining 5-mm port tied in the fifth finger and abdomen. A fascial closure was inserted percutaneously from the right lower quadrant through the abdominal wall into the peritoneal cavity under laparoscopic guidance. Then the tip of fascial closure was passed through the mesoappendix, and the tip was exteriorized through the abdominal wall. The appen-

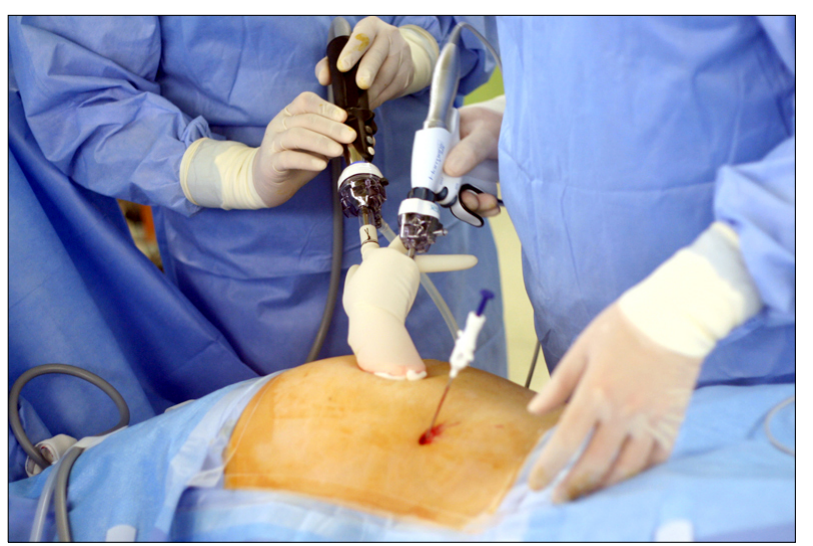

Fig. 1. Rigid 30-degree 12-mm scope, harmonic scalpel, fascia closure and surgical glove port in Group 1. dix was then hung by this fascia closure. After separating the appendix from the surrounding tissues, mesoappendix dissection and coagulation were conducted using harmonic scalpel. The appendiceal artery was ligated using an Endo Clip. First and second round loops (Laploop, Sejong Medical Co. Seoul, Korea) were placed at the base of the appendix, and a third loop was formed approximately $1 \mathrm{~cm}$ upward from the first and second ones. The resected appendix was sealed in a laparoscopic bag and removed throughout wound retractor. The umbilical fascia was closed with an absorbable vicryl 1-0 suture, and no skin suture was performed. Instead, a surgiseal (topical skin adhesive, Adhezion Biomedical, LLC, USA) was placed on the skin to close the wound.

The second group underwent surgery using another single port system (Group 2). A 3.5 5 cm vertical skin incision was made in the umbilicus, and then fascia and peritoneum were further opened. The single port, OCTO ${ }^{\mathrm{Tm}}$ PORT (Dalim, Seoul, Korea), was safely inserted into the abdominal wall. Pneumoperitoneum at $12 \mathrm{mmHg}$ was established, and the patient was put in the Trendelenburg position. A 30-degree 5-mm or 10-mm laparoscope was inserted into the TriPort. Dissecting or grasping instruments of 5-mm diameter were introduced into the port. Mesoappendix dissection and coagulation were conducted using harmonic scalpel. After dissection of the mesoappendix, a round loop was placed at the base of the appendix that was then removed through the single port. A sterile strip was used to close the wound.

The third group of patients was operated on by conventional three ports, Optical Separator (Applied Medical, Rancho Santa Margarita, CA) using harmonic scalpel (Group 3).

All patients received a single dose of prophylactic antibiotics before the operation. The operating surgeon determined the antibiotic regimen. All patients were allowed liquid diet after patients had gas out and doctors heard normal bowel sounds with a stethoscope. If patients tolerated this meal, they advanced to soft diet. Patients were discharged when able to tolerate a soft diet. No pre-incisional local blocker was used in all patients. Visual analogue scale (VAS) score was used to evaluate postoperative pain and validated by anesthesiologist for 24 hours after surgery. Nonsteroidal anti-inflammatory drugs (NSAIDs) were injected intramuscularly as needed for pain. After NSAIDs had not improved pain, intravenous opioids were used. Number of analgesics that were used for patients' pain relief was counted until their discharge from hospital.

Questionnaires regarding cosmetic results were distributed to all patients participating in this study. Questionnaires were performed from October 2011 to November 2011 via telephone interview. Patients who did not respond were contacted again. 
If this contact approach failed again, those patients were regarded as drop-outs. Two patients of 102 dropped out. Questionnaires were focused to their wound status on the year 2011. The first question involved whether the patients thought their scars healed well. The second question asked whether they were satisfied with the look of their scar. The third question entailed comparing the patients' body statuses before and after their operation. The last question involved whether they would recommend this procedure to their families or friends. The questions were evaluated using a point score: $1=$ not at all, $2=$ few, $3=$ moderate and $4=$ =very (Table 1).

Statistical analysis was performed using SPSS ${ }^{\circledR} 18.0$ (SPSS Inc., Chicago, IL) software. The significance of the differences among the means was determined by the Mann-Whitney $U$ test. A 95\% confidence interval (CI) was used to quantify the relationship between survival time and each independent factor. $p$ values $\leq 0.05$ were considered statistically significant.

\section{RESULTS}

Thirteen male and 24 female patients were included in Group

Table 1. Questionnaires regarding cosmetic results

\begin{tabular}{llllll}
\hline & \multicolumn{5}{c}{ Points } \\
\hline 1 My scar has healed well & 1 & 2 & 3 & 4 \\
2 I like the look of my scar & 1 & 2 & 3 & 4 \\
3 I am pleased with my body now more & 1 & 2 & 3 & 4 \\
than before the operation & & & & \\
4 I wound recommend this procedure to & 1 & 2 & 3 & 4 \\
families or friends & & & & \\
\hline
\end{tabular}

Points $=1$ not at all, 2 few, 3 moderate, 4 very.
1 (Gr 1); 5 male and 17 female patients in Group 2 (Gr 2); 25 male and 16 female patients in Group 3 (Gr 3). The comparison of age, height, BMI, temperature at admission, initial white blood cell (WBC), past history including diabetes mellitus, hypertension, pulmonary tuberculosis, hepatitis and operative history did not show any significant differences among the three groups. Group 1 and 2 had higher female ratio with statistical significance. Patients in group 3 weighed more than Group 1 and 2 with statistical significance (Table 2).

Operation time showed no significant difference in the three groups. No intraoperative complications were noted in the three groups. Histopathological data did not show any significant differences among the three groups. Histopathological findings of three groups are listed in Table 3. There was no conversion to conventional three ports or open appendectomy from single port appendectomy in Group 1 and 2.

The comparison of postoperative day $1 \mathrm{WBC}$, time to gas out (hours), time to start liquid diet (hours), time to start solid diet (hours), postoperative stay in hospital (hours), VAS score which was validated by anesthesiologist during first postoperative 24-hour period, and the number of IV analgesics ordered during postoperative hospital stay did not show any significant differences among the three groups (Table 4).

The evaluations of cosmetic effects are shown in Table 5 and Fig. 2. Each question was scored from 1 (dissatisfied) to 4 (satisfied) (Table 1). Outcomes regarding cosmetic effects showed that more patients of Gr 1 rated their scars well healed than did patients of Gr 2 or Gr 3 ( $p=0.001$ ) (Fig. 2). Gr 1 patients were more satisfied concerning the look of the scar than were patients in the other two groups $(p=0.000)$. Also, Gr 1 patients were more pleased with their body postoperatively than before the operation $(p=0.042)$. Overall, Gr 1 patients were

Table 2. Patient characteristics

\begin{tabular}{lcccc}
\hline \multicolumn{1}{c}{ Variable } & Group $1(\mathrm{n}=37)$ & Group 2 $(\mathrm{n}=22)$ & Group 3 $(\mathrm{n}=41)$ & $p$ value \\
\hline Number of patients & 37 & 22 & 41 & 0.532 \\
Age & $26.0 \pm 15.84$ & $27.6 \pm 13.07$ & $33.4 \pm 19.28$ & 0.235 \\
Male : Female ratio & $13: 24$ & $5: 17$ & $25: 16$ & 0.008 \\
Weight $(\mathrm{kg})$ & $52.10 \pm 14.08$ & $55.78 \pm 12.20$ & $61.44 \pm 20.33$ & 0.046 \\
Height $(\mathrm{cm})$ & $156.57 \pm 15.14$ & $160.92 \pm 11.15$ & $156.57 \pm 15.14$ & 0.336 \\
BMI (body mass index) & $20.53 \pm 3.61$ & $20.86 \pm 3.12$ & $22.46 \pm 4.52$ & 0.075 \\
Adm. Temp $\left({ }^{\circ} \mathrm{C}\right)$ & $37.10 \pm 0.60$ & $36.91 \pm 0.67$ & $36.90 \pm 0.68$ & 0.249 \\
Initial WBC $\left(\times 10^{3} / \mathrm{mm}^{3}\right)$ & $14.7 \pm 4,957.49$ & $13.9 \pm 4,197.47$ & $12.5 \pm 4,833.84$ & 0.094 \\
Past history & 7 & 2 & 7 & 0.591 \\
Operative history & 5 & 5 & 5 & 0.510 \\
\hline
\end{tabular}

Group 1 = single port appendectomy using a surgical glove; Group 2 = single port appendectomy using the TriPort system; Group 3 $=$ conventional three port appendectomy. 
Table 3. Operation and histopathological data

\begin{tabular}{lcccc}
\hline & Group $1(\mathrm{n}=37)$ & Group 2 $(\mathrm{n}=22)$ & Group 3 (n=41) & $p$ value \\
\hline Operation time (min) & $47.76 \pm 14.55$ & $54.77 \pm 12.29$ & $52.95 \pm 22.14$ & 0.150 \\
Intraoperative abscess & 0 & 0 & 0 & 0.594 \\
Intraoperative complication & 0 & 0 & 0 & 0.523 \\
Pathology & 27 & 20 & 31 & 0.535 \\
$\quad$ Suppurative & 5 & 1 & 5 & 0.512 \\
$\quad$ Exudative & 5 & 1 & 1 & 0.584 \\
Gangrenous & 0 & 0 & 4 & 0.995 \\
Perforation & & & & \\
\hline
\end{tabular}

Group 1 = single port appendectomy using a fascia closure; Group 2 = single port appendectomy using the TriPort system; Group 3 $=$ conventional three port appendectomy.

Table 4. Postoperative clinical data

\begin{tabular}{lcccc}
\hline \multicolumn{1}{c}{ Variable } & Group 1 $(\mathrm{n}=37)$ & Group 2 $(\mathrm{n}=22)$ & Group 3 $(\mathrm{n}=41)$ & $p$ value \\
\hline Postop* day 1 WBC $\left(\times 10^{3} / \mathrm{mm}^{3}\right)$ & $10.4 \pm 3,083.80$ & $9.5 \pm 2,857.93$ & $9.5 \pm 2,861.61$ & 0.387 \\
Time until gas out (hour) & $20.00 \pm 11.22$ & $24.32 \pm 12.33$ & $20.01 \pm 10.02$ & 0.438 \\
Time until to liquid diet (hour) & $30.39 \pm 9.99$ & $31.61 \pm 7.83$ & $31.67 \pm 6.366$ & 0.758 \\
Time until to soft diet (hour) & $42.99 \pm 11.80$ & $42.36 \pm 10.46$ & $41.28 \pm 5.79$ & 0.722 \\
Postop* hospital stay (hour) & $73.43 \pm 18.56$ & $68.55 \pm 14.17$ & $67.54 \pm 10.82$ & 0.308 \\
VAS score during first postop* 24-hour period & $5.08 \pm 1.71$ & $5.09 \pm 1.23$ & $5.00 \pm 1.39$ & 0.876 \\
Number of analgesics & $1.34 \pm 1.09$ & $1.09 \pm 1.44$ & $0.88 \pm 0.92$ & 0.127 \\
\hline
\end{tabular}

Group 1 = single port appendectomy using a fascia closure; Group 2 = single port appendectomy using the TriPort system; Group 3 $=$ conventional three port appendectomy. ${ }^{*}$ Postop $=$ postoperative.

Table 5. Satisfaction score

\begin{tabular}{lcccc}
\hline \multicolumn{1}{c}{ Variable } & Group 1 (n=37) & Group 2 (n=22) & Group 3 (n=41) & $p$ value \\
\hline My scar has healed well & $3.41 \pm 0.49$ & $2.68 \pm 0.82$ & $2.71 \pm 0.90$ & 0.001 \\
I like the look of my scar & $3.59 \pm 0.55$ & $2.32 \pm 0.94$ & $2.50 \pm 0.70$ & 0.000 \\
I am pleased with my body now more & $3.28 \pm 0.64$ & $2.74 \pm 0.80$ & $2.68 \pm 0.84$ & 0.042 \\
$\quad$ than before the operation & & & & 0.005 \\
I would recommend this procedure to & $3.31 \pm 0.59$ & $2.58 \pm 1.07$ & & \\
$\quad$ & & &
\end{tabular}

Group 1 = single port appendectomy using a fascia closure; Group 2 = single port appendectomy using the TriPort system; Group 3 $=$ conventional three port appendectomy.

more likely to recommend the procedure to families and friend than were the other two groups $(p=0.005)$ (Table 5, Fig. 3).

\section{DISCUSSION}

Laparoscopic appendectomy is one of the most frequently performed emergency operations. Recently, a new surgical concept, single port surgery gained attention for its cosmetic benefit, shorter hospital stay, lesser pain, and early recovery. Along with these benefits, it also raised many questions regarding its safety, usefulness, and postoperative complications. The use of single port surgery will grow in the near future for reasons driven both by patient requirements and by new surgical device development. Therefore, we would like to share our methods of conventional three-port laparoscopic appendectomy and laparoscopic single port appendectomy for acute appendicitis in order to demonstrate the advantages of single port appendectomy. Also, we compared two methods of single port surgery using 


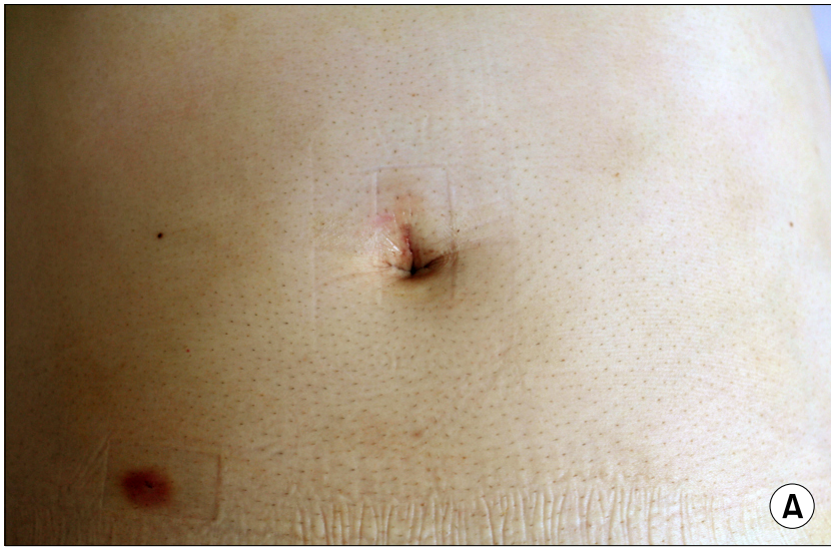

Fig. 2. (A) Postoperative view, 1 day after operation in Group 1 .

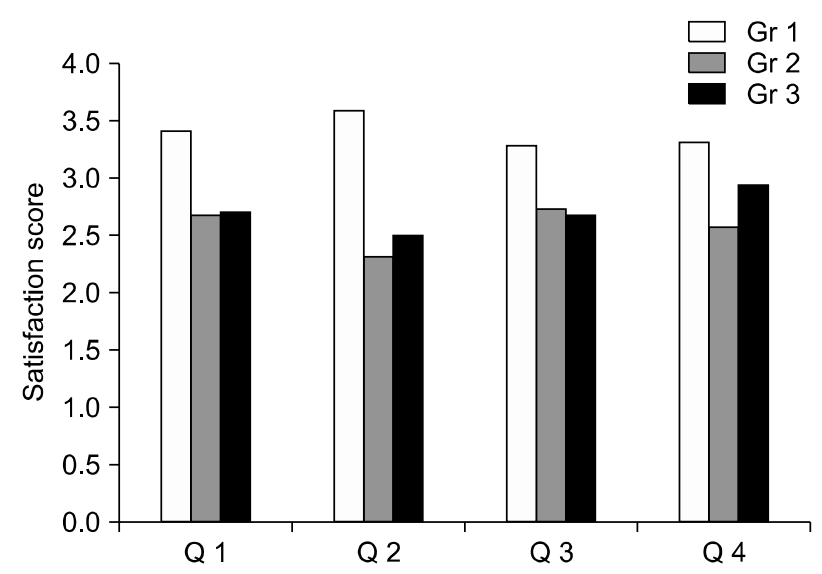

Fig. 3. Postoperative satisfaction score of three groups according to the four questions.

surgical glove and the TriPort system.

The advantages of SP have been published in other studies. ${ }^{46}$ In those articles, patients had less incisional pain, and it is believed that complications such as pain caused by the trocar can be reduced by the use of fewer trocars. It is also possible to convert a single port surgery to multiport laparoscopic surgery at any time during the surgery if necessary. A recently published study from Greece reported "significantly lower pain scores in the SILS group versus the classic laparoscopic cholecystectomy group after the first 12 hours for abdominal pain"? It seems that this result may be due to fewer incisions and reduced chance of hematoma development. In our report, a pain score was included. However, Gr 1 and Gr 2 had higher VAS scores during the first postoperative 24-hour period and higher number of analgesics taken than did Gr 3, although this was not statistically significant. Since Gr 1 and Gr 2 had longer incision of umbilicus than Gr 3, incisional pain might have re-

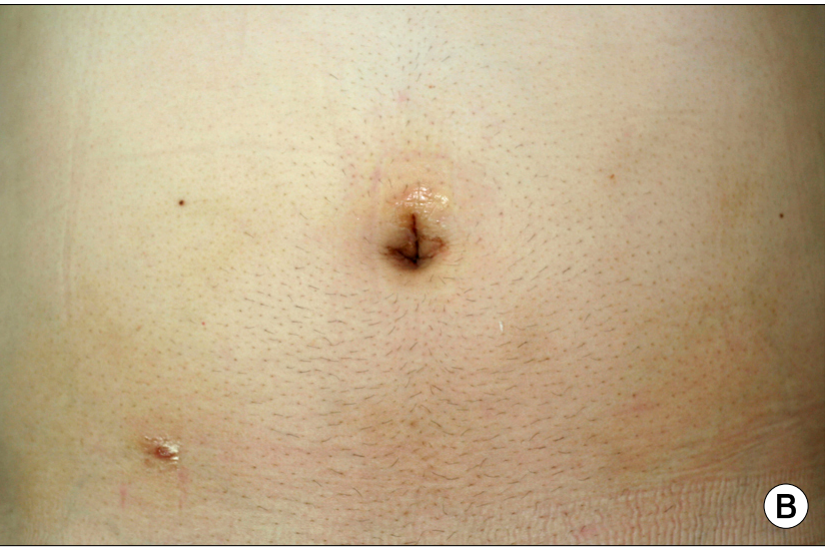

(B) Postoperative view, 7 days after operation in Group 1 .

sulted into higher pain scores in single port groups. Further studies with greater numbers of patients will be needed to prove reduced pain in laparoscopic single port surgery.

Early reports of single site appendectomy have raised concerns about the increased risk of postoperative wound infections. ${ }^{8,9}$ Koontz et al. ${ }^{10}$ reported a wound-site infection rate of 6.3\%, D'Alessio et al. ${ }^{11} 4 \%$, Meyer et al. ${ }^{12} 3.6 \%$, and Pappalepore et al. ${ }^{13} 1.5 \%$. St Peter et al. ${ }^{14}$ reported a wound infection rate of $3.3 \%$ in the single port group and $1.7 \%$ in the 3-port group. However, in the St Peter study, 3 of the 6 wound infections in the single port group occurred in the first 15 cases, suggesting the possibility that the high infection rate might be attributed to inexperienced surgeons with inadequate technique. In a similar fashion, in a large retrospective cohort of 260 children, the wound infection rate became smaller over time. ${ }^{15}$ Our report had no cases of postoperative wound infection in both Gr 1 and Gr 2 due to surgery performed by experienced laparoscopic surgeons. To minimize complications, adequate preoperative and postoperative resuscitation, appropriate perioperative intravenous antibiotics and mastery of the surgical technique will be required.

The benefit of shorter operative duration in single port surgery is still debatable. A case report by Park ${ }^{16}$ reported 40 appendectomies comparing SP and CP methods. The mean operation time of the SP group and CP group in Park's case was 75 min and $66 \mathrm{~min}$, respectively. The other retrospective study comparing 72 single site appendectomies using the standard laparoscopic approach found shorter operative times by 6 minutes on average with the single site technique. ${ }^{17}$ Another small retrospective comparative study in 35 children found the single site approach to take approximately 10 minutes longer, though this was not significant due to the small sample size. ${ }^{18}$ In this report, Gr 1 had shorter operative times than Gr 2 and 3, though 
this was not statistically significant. The difference in mean operative time implies that future studies with prospective randomized settings are needed.

Since many new port devices have been developed, choosing the right type of port device for SP surgery is the most important factor. Moreover, postoperative pain depends on the wound size, and this is one of the advantages of SP surgery. In our report, the SP method of Gr 1 using a surgical glove was compared to another SP method of Gr 2 and conventional three ports of Gr 3. Our results concluded that Gr 1 patients were more satisfied with their scars, showing overall higher satisfaction scores than Gr 2 and 3 patients $(p<0.05)$. The reason for higher satisfaction score in Gr 1 is due to the barely visible postoperative scar compared to the other two groups. The benefits of SP surgery using a surgical glove were mentioned in a previous article. ${ }^{19}$ The wound retractor allows an oval-shaped, free hole to be obtained with a $1.5-\mathrm{cm}$ skin incision, which facilitates smooth movement of instruments and easy position changes. In addition to choosing the type of port device, the number of port insertions, port placement sites, and port sizes are also considered important factors for postoperative pain, morbidity, and cosmetic results of SP. We minimized surgical trauma using a transabdominal fascial closure instead of a second port. We successfully suspended the appendix and cecum from the surrounding tissues using only the fascial closure. This method might have prevented possible complications and prolonged operating times. Our data support this hypothesis by showing shorter operative times in Gr 1, even though it did not attain statistical significance. Other review also demonstrated the safety and feasibility of the fascial closure in laparoscopic surgery. ${ }^{20} \mathrm{SP}$ using fascial closure could be regarded as a single port method, since fascial closure left barely visible wound scar postoperatively.

As single port surgery becomes more popular, various studies have focused on its cosmetic results, but not many of them have shown the results quantitatively. Over the past years, several scales including the Vancouver Scar Scale (VSS), Manchester Scar Scale (MSS), Patient and Observer Scar Assessment Scale (POSAS), Visual Analog Scale (VAS), and Stony Brook Scar Evaluation Scale (SBSES) have been used to evaluate scar appearance in burn patients. This study adopted to a previously published article and created new type of questions from it. ${ }^{21}$

Our study assessed patient satisfaction with their wound status on the year 2011 using a questionnaire. The questionnaire was scored from 0 (dissatisfied) to 4 (satisfied). Group 1 had a statistically significantly higher overall satisfaction score than did the other two groups for all questions. This result shows that there is a certain advantage of performing the single port surgery compared to the standard procedure. A comparative study with 87 patients $^{22}$ supports our result by reporting that patients were more satisfied with SP than CP $(p<0.05)$. The data generated by our telephone interviews allowed statistically significant and meaningful conclusions to be drawn regarding patient satisfaction and cosmetic appearance.

Our study has several limitations. All three groups used different types of antibiotics, and each group was operated on by different surgeons, which might affect results regarding complications and satisfaction scores. To overcome these limitations, one regimen of antibiotics and one surgeon should be used for all patients. Proportion of women and low BMI patients were higher in Gr 1 and Gr 2 than Gr 3. As a result, single port groups were easier to perform surgery than conventional three port group. Surgeons might have chosen their modes of surgery according to gender and weight of patients. To minimize selection bias, randomized controlled trials should be designed in the future.

\section{CONCLUSION}

In conclusion, laparoscopic single port appendectomy using a surgical glove is a feasible and safe procedure and shows no differences in postoperative clinical data compared to a conventional three-trocar technique. Furthermore, this method results in better satisfaction than the other two groups, illustrating its cosmetic improvement.

\section{REFERENCES}

1) Ben-David K, Sarosi GA Jr. Appendicitis. In: Feldman M, Friedman LS, Brandt LJ, editors. Sleisenger \& Fordtran's Gastrointestinal and Liver Disease. Vol 2. 9th ed. Philadelphia, PA: Saunders Elsevier; 2010. p. 2599-2612.

2) Semm K. Endoscopic appendectomy. Endoscopy 1983;15:59-64.

3) Esposito C. One-trocar appendectomy in pediatric surgery. Surg Endosc 1998;12:177-178.

4) Park J, Kwak H, Kim SG, Lee S. Single-port laparoscopic appendectomy: comparison with conventional laparoscopic appendectomy. J Laparoendosc Adv Surg Tech A 2012;22: 142-145.

5) Cho MS, Min BS, Hong YK, Lee WJ. Single-site versus conventional laparoscopic appendectomy: comparison of short-term operative outcomes. Surg Endosc 2011;25:36-40.

6) Roberts KE. True single-port appendectomy: first experience with the "puppeteer technique". Surg Endosc 2009;23:1825-1830.

7) Tsimoyiannis EC, Tsimogiannis KE, Pappas-Gogos G, et al. Different pain scores in single transumbilical incision laparoscopic choecystectomy versus classic laparoscopic chol- 
ecystectomy: a randomized controlled trial. Surg Endosc 2010; 24:1842-1848

8) Ponsky TA, Diluciano J, Chwals W. Early experience with single-port laparoscopic surgery in children. J LaparoendoscAdv Surg Tech 2009;19:551-553.

9) Garey CL, Laituri CA, Ostlie DJ. Single incision laparoscopic surgery in children: one institution's experience. J Pediatr Surg 2011;46:904-907.

10) Koontz CA, Smith LA, Burkholder HS. Video-assisted transumbilical appendectomy in children. J Ped Surg 2006;41:710-712.

11) D'Alessio A, Piro E, Tadini B, Beretta F. One-trocar transumbilical laparoscopic-assisted appendectomy in children: our experience. Eur J Pediatr Surg 2002;12:24-27.

12) Meyer A, Preuss $M$, Roesler $S$, Lainka $M$, Omlor G. Transumbilical laparoscopic-assisted "one-trocar" appendectomy-TULAA, as alternative operation method in the treatment of appendicitis. Zentralbl Chir 2004;129:391-395.

13) Pappalepore N, Tursini S, Marino N, Lisi G, LelliChiesa P. Transumbilical laparoscopic-assisted appendectomy (TULAA): A safe and useful alternative for uncomplicated appendicitis. Eur J Pediatr Surg 2002;12:383-386.

14) St Peter SD, Adibe OO, Juang D, et al. Single incision versus standard 3-port laparoscopic appendectomy: a prospective randomized trial. Ann Surg 2011;254:586-590.

15) Muensterer OJ, PugaNougues C, Adibe O. Appendectomy using single-incision pediatric endosurgery for acute and perforated appendicitis. Surg Endosc 2010;24:3201-3204.

16) Park JH, Hyun $\mathrm{KH}$, Park $\mathrm{CH}$, et al. Laparoscopic vs transumbilical single-port laparoscopic appendectomy: results of prospective randomized trial. J Korean Sur Soc 2010;78:213-218.

17) Visnjic S. Transumbilical laparoscopically assisted appendectomy in children: high-tech low-budget surgery. Surg Endosc 2008;22:1667-1671.

18) Lee J, Baek J, Kim W. Laparoscopic transumbilical single-port appendectomy: initial experience and comparison with three-port appendectomy. Surg Laparosc Endosc Percutan Tech 2010;20: 100-103.

19) Hayashi M, Asakuma M, Komeda K, et al. Effectiveness of a surgical glove port for single port surgery. World J Surg 2010;34:2487-2489.

20) James E, Carter A. New technique of fascial closure for laparoscopic incisions. J Laparoendosc Surg 1994;4:143-148.

21) Kapischke M, Friedrich F, Hedderich J, Schulz T, Caliebe A. Laparoscopic versus open appendectomy--quality of life 7 years after surgery. Langenbecks Arch Surg 2011;396:69-75.

22) Vilallonga R, Barbaros U, Nada A, et al. Single-port transumbilical laparoscopic appendectomy: a preliminary multicentric comparative study in 87 patients with acute appendicitis. Minim Invasive Surg 2012;2012:492409. 